DOI: https://doi.org/10.14311/TPFM.2018.032

\title{
EDDY STRUCTURE AND PATTERNS IN THE OCEANS AND ATMOSPHERES
}

\author{
J. M. Redondo ${ }^{1}$, S. Karimova ${ }^{1,2}$ \\ ${ }^{1}$ Department of Physics, Universidad Politecnica de Cataluña, Barcelona Tech., Campus \\ Nord B5, E-08034 Barcelona, Spain \\ ${ }^{2}$ Department of Astrophysics, Geophysics, Oceanography, GHER, University of Liege, S, \\ Liege, Belgium
}

\begin{abstract}
The use of thematic maps derived from a combination of satellite sensors and images are validated between different methods and with on board observations, the topology of the detected features, such as spiral eddies [1, 2], fronts, Langmuir cells, etc.. May be even be used to predict diffusion and local pollution. It is also important to couple satellite information to the multiscale information available, which may be used to infer the turbulent parametrizations needed to predict pollutant and tracer dispersion in the ocean surface. In such a manner, more sophisticated data analysis such as the evaluation of integral length scales or local fractal dimensions of the sea surface appearance, and the detailed information of the position and sizes of the mesoscale dominant eddies provides very useful information on the sub-mesoscale ocean turbulence. The satellite-borne SAR and ASAR seems to be an excellent system not only to detect man-made oil spills and tension active slicks but it also detects dynamic features and the ocean eddies of different sizes. The study of the topology of the regions of different rugosity of the ocean, separating the vortical, elliptical, regions as well as the hyperbolic shear dominated areas, is also a convenient tool. The eddy structures of the Western Mediterranean were also investigated at different resolutions also by means of thermal images and altimetry data. A comparison of 1489 anticyclonic and 782 cyclonic eddy structures identified in the sea surface, to ASAR observations was performed. The (SST) imagery on an analysis of a 3-year period shows that only large anticyclonic eddies exceeding $70 \mathrm{~km}$ in diameter are long lived. This analysis, also applied to SAR and in advanced tracking of STT sequences, allows CPIV to extract velocity and vorticity local fields and relate spiral structure to vortex size. In the NW Mediterranean cyclonic eddies depend further on bathymetric effects. Due to the lack of resolution small spatial and temporal scales of such eddies and their non-geostrophic nature, it is difficult to compare basins of different size.
\end{abstract}

Keywords: Spirals, Sub Mesoscale Eddies, Ocean Vortices, Rossby, SAR

\section{Introduction}

A better understanding of the effects of the inverse energy cascade in 2D turbulence is important for applications of the theory in geophysical flows, which are often dominated by large vortices. Evidence for or against logarithmic corrections or scaling is not definitive and is unlikely to be resolved in the near future owing to the difficulties differentiating effects and insensitive logarithmic scaling. which is also reflected in the main Tidal and Inertial component. The many forcings taking place in the ocean surface produce a wealth of topologically coherent features [ 2, 3 ]. Here we compare and discuss different types of detected eddies and spiral features in the atmosphere and ocean. The size of the ocean basin, of course places a limit to the topology of the flows, but also the distance to the coastline, to a bathymetric or geographical feature, etc. When buoyancy and the earth rotation are dominant, there is an equilibrium horizontal scale measured following the geopotential lines: The Rossby deformation radius, which may be interpreted as the horizontal scale where Coriolis force is in equilibrium with the stratification induced gravitational force, or buoyancy, this scale limits the eventual growth of the eddies to scales of the order of the basin. In the Atmosphere LR is much larger than in the Ocean, where often the relevant vertical scale is the characteristic scale of the depth of the thermocline [3, 4]. If the scale of the vortices is greater than the calculated value of LR they are unstable and are broken into smaller ones, mostly because the 
excess angular momentum induced by the earth rotation may not be balanced by pressure due to buoyancy.

The largest vorticity generation in the Atmosphere takes place in the North and South Poles, but zonal winds may also induce stratospheric vorticity. In the Tropopause the variations of vertical vorticity with latitude generates Rossby Waves, that are responsible of most of the Cyclone / Anticyclone Easterly drift in the northern hemisphere. This dominant Anti Cyclonic flow, or positive vorticity source in the northern hemisphere is dominant. In ocean the local vorticity at small scale, may have multiple sources, vorticity generated by the bathymetrical particularities of the sea bottom as well as the coastal friction in the Mediterranean Sea this seems to destabilize further the large vortices drifting South Westerly due to the Liguro-Provenzal current. [1/3] The basic effect of Conservation of Potential Vorticity is also an important local driving force. We describe some of the techniques used to investigate the topology and shapes emerging in the atmosphere and in the ocean surface, then we limit ourselves to the Western Mediterranean and to spiral shapes, we finally compare different decaying scaling laws and present the conclusions.
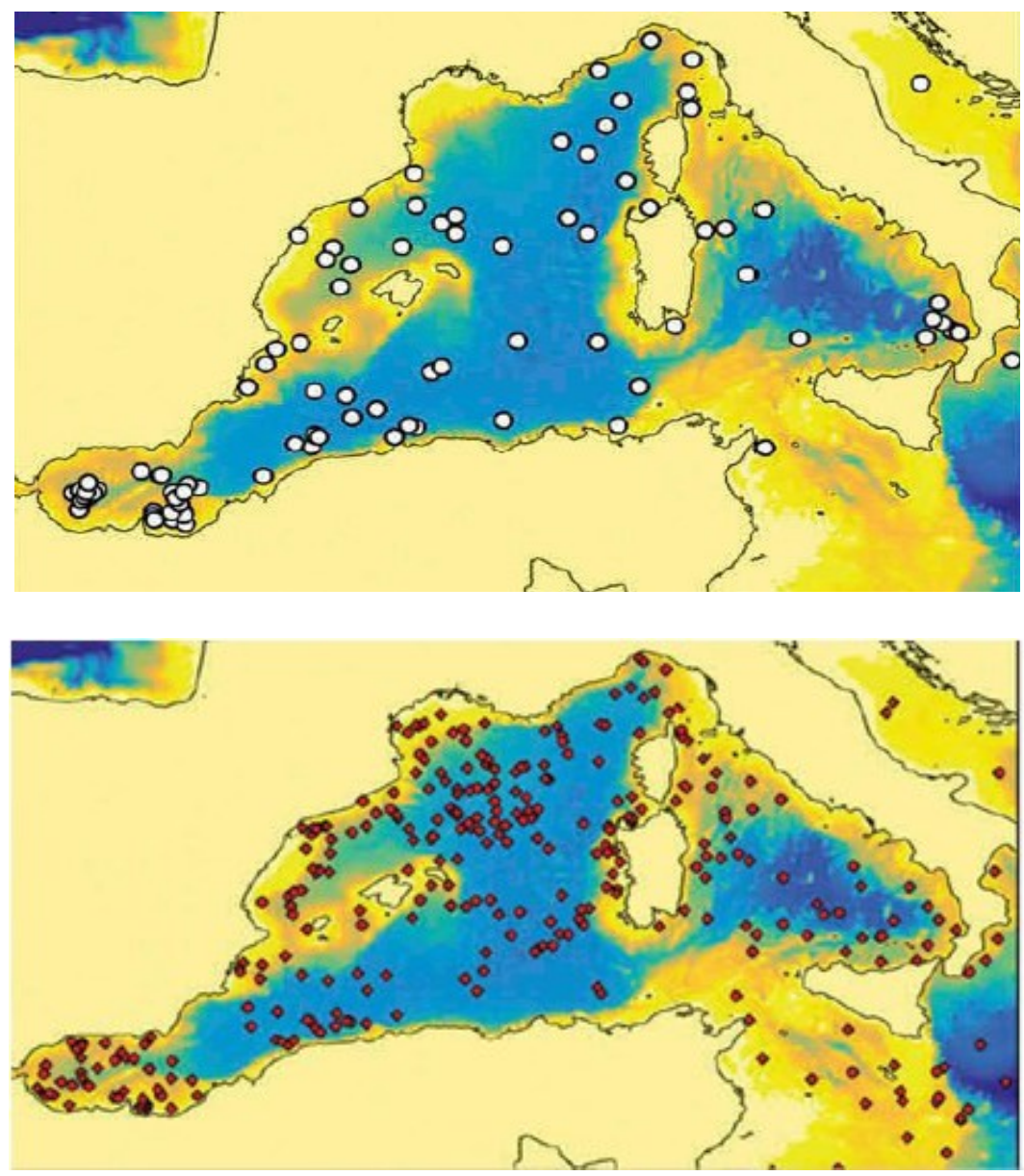

Figure 1: Different types of eddies in the Mediterranean Sea (top) Cyclonic, (bottom) Anticyclonic [20]

\section{Eddy Probability Distribution Functions and Identification of Spirals}

The observed hyperbolic type of vortex distribution, is associated with Zipf's Law and also indicates a turbulent type power law spectrum, most of the detected features by SAR and other sensors [9, 16] have either Rayleigh of Weibull distributions as a function of size, the anisotropic eddies were assigned an average length as $\mathrm{L} \sim 2$ (a b) ${ }^{1 / 2}$.

Turbulent diffusion in the Ocean is important and directly related to the distribution of energy among the different scales, there is a range of different inertial type of scaling, but the main hypothesis of local 
equilibria is not normally assured, and even less so in non-homogeneous flows, The large Lagrangian buoys also provide direct information, together with cruise measurements transversing a satellite detected eddy, but further detailed small scale information of the surface and sub/surface velocity is needed. Soon hundreds of small and inexpensive tracer (GPS) Lagrangian buoys may be used also near an accident to aid the predictions of coastal currents. Diffusion in the ocean exists on different space scales: from molecular level (molecular viscosity and diffusion) to oceanic turbulent processes (from centimetres and metres vertical scale to tens and hundreds of kilometres of horizontal scale). Turbulent diffusion is directly linked to the dominant features and instabilities [3-6]. After an initial Ballistic phase, the nonlineal processes begin to dominate and new dynamic scales appear, the maximum concentrations of the tracers shows a non linear decay The study of the turbulent diffusion is based on two methods. The first is Lagrangian (monitoring and numerical analysis of the motion of the particles or tracers) and the second one is Eulerian with a characterization of the spatial distribution of the velocities, correlations and energy spectral characteristics that may affect locally the turbulent diffusion $[7,8]$.

These coherent structures show clear traces in satellite imagery, and originate in the average vorticity field localized intense concentrations, which merge, partially axisymmetrize, and develop into welldefined vortices that persist for time scales much longer than the enstrophy transfer time $Z$. The analysis of the structures present in the SAR images requires a good understanding of the diffusion of electromagnetic pulses emitted by the moving sensor to image the surface of the ocean. [8,9] Backscattered energy is considered in the frequency domain for the azimuthal direction using Doppler spectrum, and in the time domain for the radial direction. The state of the surface observed depends also on local atmospheric and ocean conditions considered, which vary in time and space between a smooth surface (black SAR images) and a surface excessively rough or with considerably floating debry, which due to the backscattered radar appears white in SAR images. The assumption of gradual roughness at various scales can be seen at the ocean surface. The so-called small-scale Bragg waves form also in response to the force of the wind. The backscatter is due to the component of the wave spectrum that resonates with the wavelength of the radar. Using a systematic analysis of satellite images, there is a method of calculation of the average eddy diffusivity from a sequence of SAR images, using dimensional analysis and the local scales measured as integrals of the SAR reflectivity spatial correlations, here the local influences of the wind and the currents are important. Nevertheless, on the long run, horizontal directions will average out so using a single integral length scale defined in will be enough together with the inertial frequency. A method involving the multi-fractal dimension measurements [9, 14] is much more elaborated and seems to have a better theoretical justification in the sense that it is possible that different concentrations showing different fractal dimensions may be due to different levels of intermittency and thus different spectra, which are not necessarily inertial nor in equilibrium.

To relate spatial topological features detected by SAR to the local diffusivity, which depends on Waves, wind and local bathymetry as shown by [16, 17]. Then reference plots of features such as the maximum fractal dimension with the integral of the fractal dimension over all possible intensity levels of SAR can be used to predict the behaviour of the oil spills. Such curves would grow monotonically in time and may give us information, taking account on the sea surface local weather, on how long ago was a particular oil spill released onto the sea. The topological structure may also help us to distinguish between oil seeps from the ocean bottom (more distributed) and oil spills from ships (elongated) $[17,18]$
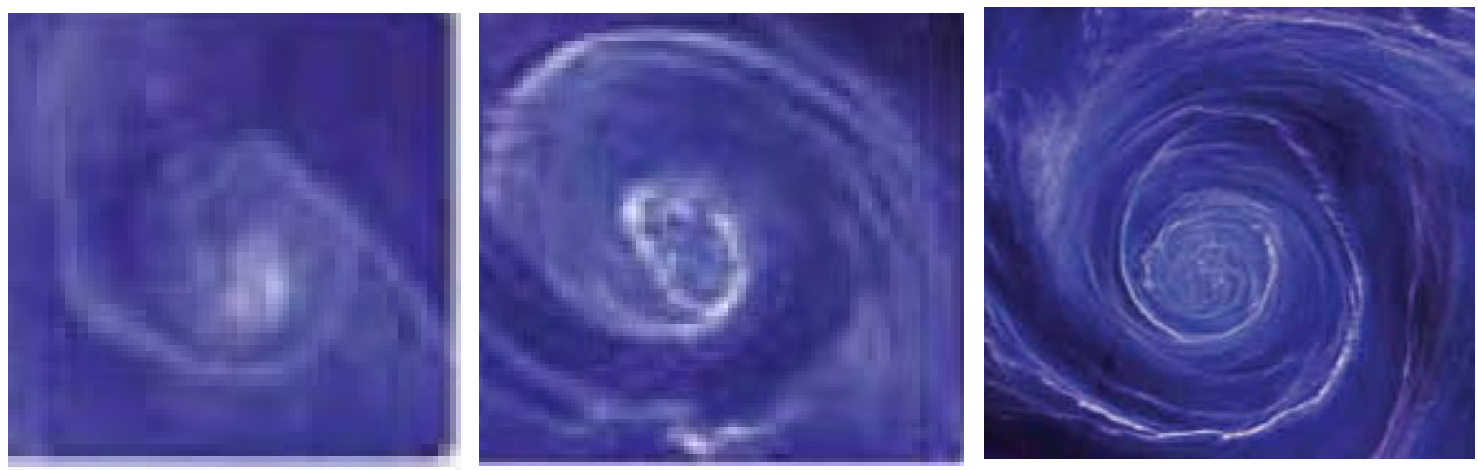

Figure 2: Spiral eddies of different sizes in the Black Sea [8, 20] Spiral arms are clearly observed. 
The extension of the 2D decaying turbulence picture to the much more complex ocean surface eddy $\Omega \omega$ model is by no means straight forward [11-13], the same is true in the atmosphere or in planetary atmospheres, where many geostrophic and barotropic and baroclinic, local and external enstrophy sources and sinks are present. This idealized model of turbulence and coherent structure behaviour, with additional 3D influences may still be of some use when interpreting the probability distribution functions in size as shown both in Figures 2, 3 and 5. If we fit a logarithmic spiral to spiral eddies of different size as shown in Figure 2, we see that the smaller the eddy the lesser the number turns of a well defined arm, this means that the function $\Theta(Z, A)$ is inversely proportional to the size of the vortex, this also needs to be checked for different conditions and local values of Enstrophy $Z=1 / 2 \omega^{2}$ especially to atmospheres and planetary oceans and atmospheres. Notice here that $A=\pi r^{2}$ The forcing scales are very wide and have local sinks and sources. If we write an equation for the Archimedes spiral fitted in any of the observations, like those shown in Figure 2. With the dependence between the radius of the arm and the angle as

$$
r(Z, A, \beta)=C(\beta) e^{\Theta(Z, A)},
$$

the role of the local enstrophy will in general not only affect the size and shape of the vortex or spiral eddy, but will also affect its evolution in time $t$, in order to compare the real behaviour of detected spiral features, we can assume that the ocean turbulence is in a state of free 2D turbulence decay in the simplest unforced equilibrium with the irrotational and incompressibility fluid conditions, then it is easy to show $[11,12]$ that if the vortex translational speed does not depend on the vortex size $A$, or on its enstrophy $Z$, Then the total area (vorticaly active) covered by a number of freely decaying vortices in the ocean surface $n$ will be

$$
A=A(Z, r, \beta)=C \sum_{1}^{n} A_{n}
$$

And the time dependence is derived from the simple assumption of evolution as

$$
\begin{aligned}
& \frac{\partial A}{\partial t}=\frac{1}{A^{2}} \\
& A(t) \approx t^{\frac{\beta}{2}}
\end{aligned}
$$

So that

And for the number of vortices with total area $\mathrm{A}(\mathrm{t})$

$$
n(t) \approx t^{-\beta}
$$

So while the number of decaying vortices in an unforced generalized simple ocean decreases, their total area increases. An easy check if these idealised conditions hold is to see the deviation of the product $A(t) . n(t)$ just evolves as the square root of time, i.e. $A . n=t^{1 / 2}$. The $2 \mathrm{D}$ turbulence decay law with the above hypothesis may be invoked to find $\beta=2 / 3$. Then also $n(A) \approx t^{-\beta} A^{-1}$.

The natural eddy turnover time is related to the Enstrophy as $T_{\omega} \approx Z^{-\frac{1}{2}}=\varpi^{-1}$

From Figure 2 the larger spiral eddies have a tighter wind up of arms, so the relationship between the angle and the Area exhibits a decaying power law, dimensional analysis is needed to choose among the possible additional dependences that link the topological or shape aspects to the dynamical aspects, in the same way the local enstrophy will also affect the arms of the spiral eddies. Of course the role of forcing events, both by current shear of by atmospheric forcing alters the decay process, but observations of time lapse indicate the intermittency of both forcing an decaying vortical events. The largest vortices are not stable mainly due to several reasons: The stability of the vortices depends on the Rossby deformation radius $L_{R}$ determined in terms of buoyancy parameterized through the Brunt Vaisalla frequency and also of the inertial frequency, given by the Coriolis parameter, $f$

$$
f=2 \Omega \cos \theta
$$



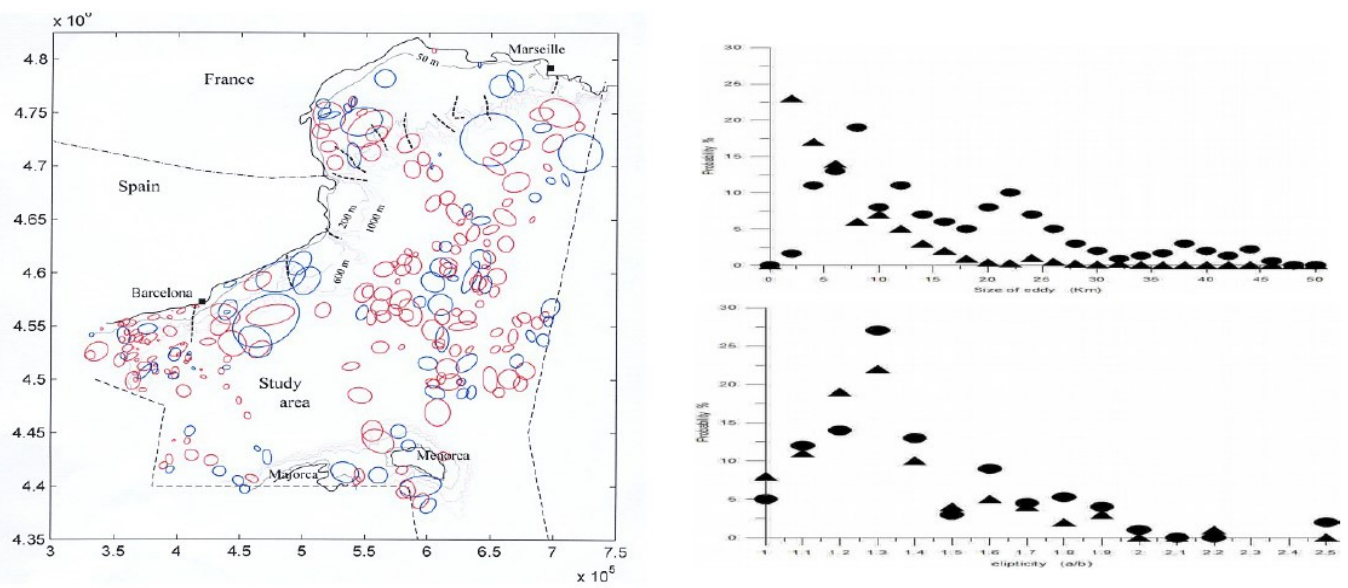

Figure 3: Eddy distribution of Clean Seas results $[1,17,18]$

The calculated values of the Rossby deformation radio LR confirm the existence of larger stable eddies in summer than in winter in the NW Mediterranean. Other areas have slightly different ranges, in particular the Rhone area agrees quite well with the observed sizes in $[8,17,20]$

The Rossby deformation radius of the typical maximum size of the vortices vary from zone to zone and from season to season, In the area covered by Figure 3 is of the order of $20 \mathrm{~km}$, an area A of a stable vortices is near $300 \mathrm{~km}^{2}$. A value of diameter of the vortices detected in cruises of $13 \mathrm{~km}$, and the statistical analysis with thermal IR Satellite images $[8,20]$ validates this observation. This dependence will change with thermal seasonal changes and to changes in the sea surface heat flux balance. Concerning the shape of the vortices detected in coastal waters shown in Figure 3 between 1996 and 2000. Most of them $(40 \%)$ have an elliptical accused form and the relation between the principal and secondary axis varied between 1.1 and 1.6 (Figure 3), that also supports that the elliptical vortices are more stable that circular ones. In the case of the more general analysis of all areas, this particularity is not so clear $[9,20,21]$.

The extension of most of the SAR detected vortices (63\%) is less than 100 square kilometres, $33 \%$ of vortices occupy an area between 100 and $500 \mathrm{~km}^{2}$ and only $4 \%$ of the vortices possess a larger area between 500 and $1200 \mathrm{~km}^{2}$. About $93 \%$ of vortices have a diameter less than $20 \mathrm{~km}$. Most of the vortices (79 \%) have an ellipticity (relation between large and small diameter) near 1.12-1.63 near Barcelona due to bathymetry, there is a preferred orientation of the vortices is direction between NW and NE. Figure 3 and Table 1 shows the size distribution of these eddies in different areas.

Table 1. Size ranges of detected eddies $[8,22]$

\begin{tabular}{lcrcccccc}
\hline & $\begin{array}{c}\text { Range of } \\
\text { diameters } \\
(\mathrm{km})\end{array}$ & $\begin{array}{c}\text { Number } \\
\text { of } \mathrm{C} \\
\text { eddies }\end{array}$ & $\begin{array}{c}\text { Number } \\
\text { of } \mathrm{AC} \\
\text { eddies }\end{array}$ & $\begin{array}{c}\text { Percentage } \\
\text { of C eddies } \\
(\%)\end{array}$ & $\begin{array}{c}\text { Mean } \\
\text { diameter of } \\
\text { C eddies }\end{array}$ & $\begin{array}{c}\text { Mean } \\
\text { diameter of } \\
\text { AC eddies }\end{array}$ & $\begin{array}{c}\text { Rossby } \\
\text { radius } \\
(\mathrm{km})\end{array}$ & $\begin{array}{c}\text { Transition } \\
\text { scale }(\mathrm{km})\end{array}$ \\
\hline Baltic & $1-59$ & 4660 & 283 & 94.3 & 6.6 & 11.6 & $1.3-7^{\text {a }}$ & - \\
Black & $20-113$ & 790 & 1198 & 39.7 & 35.0 & 51.3 & $15-20^{\text {b }}$ & 40 \\
North & $10-60$ & 2145 & 257 & 89.3 & 24.2 & 34.1 & $10-15^{\mathrm{c}}$ & 50 \\
Western & $20-170$ & 347 & 113 & 75.4 & 31.4 & 65.8 & $10-18^{\mathrm{d}}$ & $60-80$ \\
$\quad$ Mediterranean & & & & & & & & \\
\hline
\end{tabular}

we concentrate our attention on mesoscale and submesoscale circulation features of the LiguroProvençal Basin captured by satellite radiometer, spectroradiometer, and radar images. Using such a dataset makes it possible to observe the circulation features from a wide spatial range, from the basin scale through mesoscale to the scales of a few kilometres. Mesoscale features in this study are being mostly observed with thermal infrared imagery retrieved by AVHRR and AATSR sensors. Special attention in the work was paid to an analysis of the data coming from a geostationary satellite, namely ones provided by SEVIRI. Due to their uniquely high temporal resolution, such imagery allows observing circulation features in their evolution. 
During the winter blooming events, surface circulation at meso- to submesoscales in the region of interest was additionally highlighted by images obtained in the visible range. Full spatial resolution images provided by Envisat MERIS, Sentinel-2 MSI, and Landsat TM/ETM+/OLI made the greatest contribution to this part. The smallest scales (namely submesoscale) are being observed with synthetic aperture radar (SAR) imagery provided by Envisat ASAR and Sentinel-1 SAR. During an analysis of SAR images, it was noted that there was strikingly great amount of biogenic surfactants on the water surface in the region of interest. Apparently, low biological productivity typical for the Western Mediterranean ecosystem is not a limiting factor for the formation of surfactant films seen in SAR imagery. It is important to consider the presence of surfactants, because they behave as good tracers of surface currents when these are locally aligned with the surface flow, Langmuir cells are also an important factor in aiding the visualization of the surface eddies [20,21]. As discussed above, when the inner spiral arms of an eddy appear Black it means that tensioactive substances such as antropogenical oil spills of biogenical plankton slicks are converging in these streaks, one of the conditions for black eddies or spirals to be observed in SAR is a moderate wind. On the other hand, and normally when ocean surface wind is stronger, white eddies appear when there is a substantial debris concentration, and wake roughness if drawn into streaks within the spiral arms. A careful analysis of local conditions may also be useful for pollution control further than discussed in $[17,18,20]$.

\section{Ocean Vortex Dynamics and Observations}
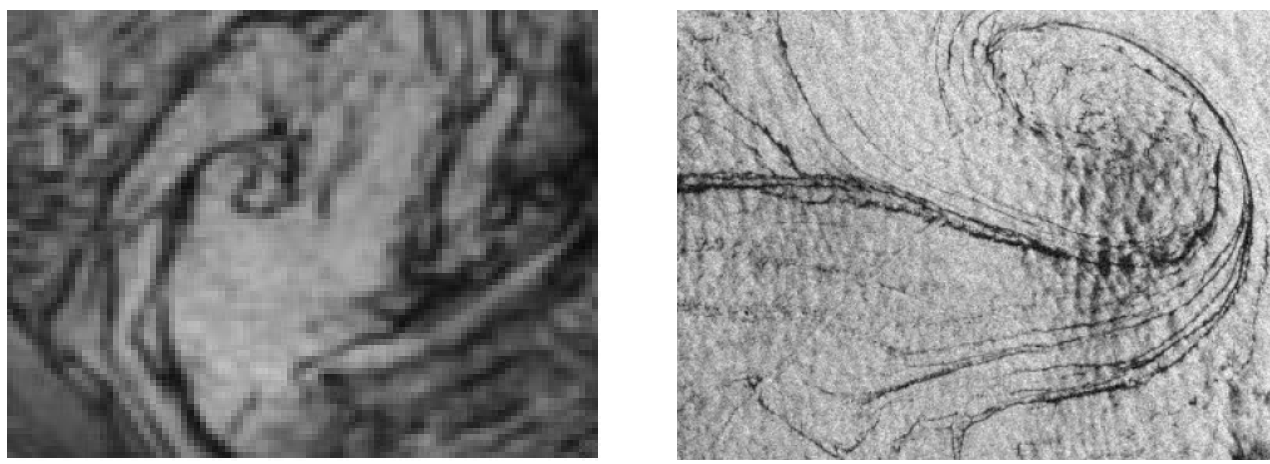

Figure 4: Typical, "Black” marked Spiral Eddy (left). Vortex Dipole detected by SAR (right) [9, 10]

The PDF of the basic instabilities are different if they are analysed at scales smaller, or greater than the Rossby Deformation Radius scale LR. The Results show the ability to identify different SAR signatures and provide calibrations for the different configurations of vortices, (round or elliptical), fronts, spirals, Langmuir cells, oil spills and tensioactive slicks are all relevant and eventually allow some predictions of the self-similar structure of the environmental rotating/stratified turbulence. Such complex coastal field dependent behavior is strongly influenced by stratification and rotation of the turbulence [2, 12]; non homogeneous, and non local spectra are observed only in the range smaller than the local LR.

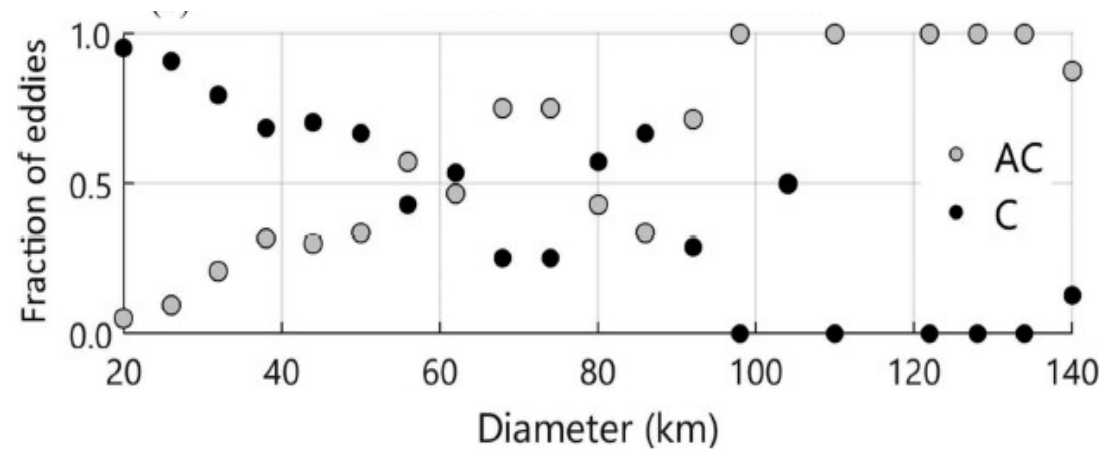

Figure 5: Cyclonic (C)and Anticyclonic (AC) Eddies "Black" or White marked Spiral Eddies Vortex

Dipoles are also included $[9,10,20]$. The observations correspond to the Mediterranean Sea.

Measures of diffusivity from buoy or tracer experiments are used to calibrate the behavior of different tracers and pollutants. Using different polarization and intensity levels from satellite imagery, we may 
distinguish between natural and man-made sea surface features due to their distinct self-similar and fractal appearance as a function of spill parameters [4, 17], environmental conditions and history of both oil release and coupled Atmosphere-Ocean interface weather conditions.in 3D e GlobCurrent project, and the data by reanalysis. Special attention is being paid to the performance of different datasets in manifesting largest mesoscale eddies of the region of interest. As a result of the comparison performed, the general scheme of surface circulation in the region of interest was updated. the new scheme of [20, 21] concerns the number of cyclonic gyres present within the field of surface currents.

Even though the region of interest has a low mean eddy kinetic energy, the additional analysis of the images of the Liguro-Provençal Basin show a surprisingly high eddy activity within submesoscale and mesoscale features. But the size of eddies in this area were generally smaller than that in the southern part of the Western Mediterranean. The observations show that the main contributors to generation of observed mesoscale vortical structures are (i) the instability of the main currents in the region of interest and especially frontal instability at the Liguro-Provençal front and (ii) instabilities caused by the coastline inhomogeneity, especially in the eastern part of the Basin. Submesoscale eddy activity seems to be developed to its full extent during the periods when the mesoscale activity in the region of interest. The measurements with advanced vorticity-Stream function patterns thanks to buoy tracking. The study of the fractal and self-similar structure of the turbulence is performed at different levels of SAR intensity and Polarization is also different. Depending on the surface wind and wave level, and also on the fetch and the bathymetry Figure 6 shows the new tools available to study the topology of the Atmosphere and Ocean.
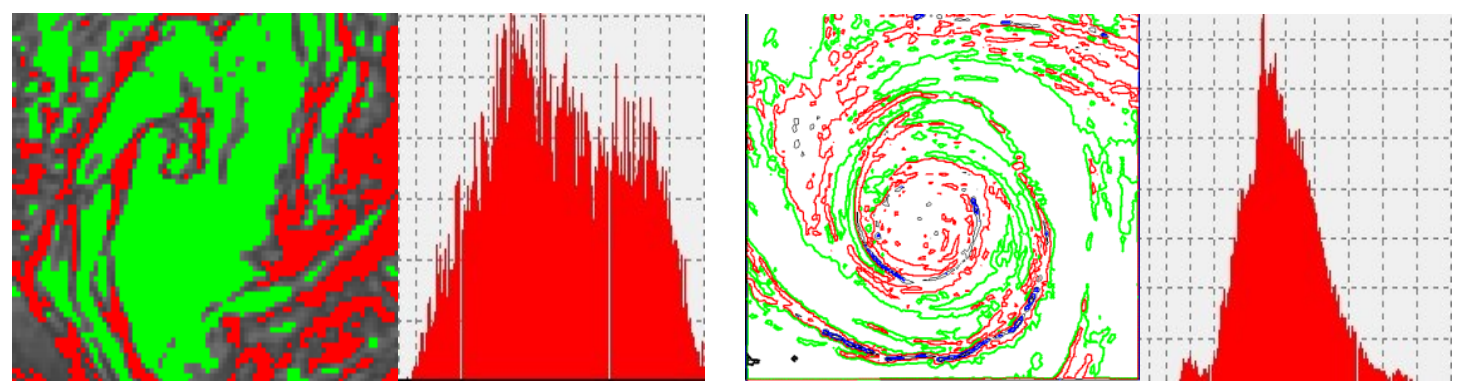

Figure 6: SAR Spiral Eddy Scaling Analysis (left) [16, 17]. Vortex detected by ESS analysed with ImaCalc (right) [20, 21]

New measurements such as the role of the distance from the Thalwegs, the Spiral topology, its arm structure and their anisotropic characteristic parameters are function of the resolution of vortical features and their scale, also change $[1,2]$ in time and space. Further analysis is needed also when comparing eddy structure derived from altimetry, because the insensitivity of surface height to Cyclonic or Anticyclonic eddies is an important source of errors.

Acknowledgements

This work was supported by the Spanish Ministry and the Universitat Politecnica de Catalunya (FTN2001-2220, ESP2005-07551, RYC-2003-005700). Authors also acknowledge the (ENV4-CT96-0334) and (FP7-PEOPLE-COFUND-BelPD) European Union Projects and the ESA (AO-ID C1P.2240).

\section{References}

[1] Jolly, G. W., Mangin, A., Cauneau, F., Calatuyud, M., Barale, V., Snaith, H. M., Rud, O., Ishii, M., Gade, M., Redondo, J. M., and Platonov, A.: The Clean Seas Project Final Report (ENV4-CT960334), Ed. DG XII/D, Brusselas, (2000).

[2] Munk, W., Armi, L., Fischer, K., and Zachariasen, F.: Spirals on the Sea, P. Roy. Soc. A-Math. Phy., vol. 456, 1217-1280, 2000 Redondo, J.M.: Vortices, Streaks and Fronts in Ocean/Atmosphere interfaces. Nonlinear processes in oceanic and atmospheric flows, vol. NLOA2016, no. 1: (2016) pp. 47-49.

[3] Carrillo, A., Sanchez, M. A., Platonov, A., and Redondo, J. M.: Coastal and Interfacial Mixing. Laboratory Experiments and Satellite Observations, Phys. Chem. Earth B, 26, 305-311, (2001).

[4] Diez, M., Bezerra, M. O., Mosso, C., Castilla, R., and Redondo, J. M. Experimental measurements and diffusion in harbor and coastal areas, Il Nuovo Cimento C, 31, 843--859. (2008). 
[5] Flexas, M. M., Van Heijst, G. J. F., and Trieling, R. R.: The behaviour of jet currents over a continental slope topography with a possible application to the Northern Current, J. Phys. Oceanogr., 35, 790-810, (2005).

[6] Gade, M. and Redondo, J. M.: Marine pollution in European coastal waters monitored by the ERS-2 SAR: a comprehensive statistical analysis, IGARSS 99, Hamburg, vol. III 1637-1639, 308-312, (1999).

[7] Redondo, J. M.: Turbulent mixing in the Atmosphere and Ocean, Fluid Physics, World Scientific, New York, 584-597, (1994).

[8] Karimova S.: Observation of the surface circulation of the Mediterranean Sea from space. In ESA Living Planet Symposium 20161 SP-720, (2016).

[9] Redondo, J. M. and Platonov, A. K.: Aplicacion de las imagenes SAR en el estudio de la dinamica y de la polucion del mar Mediterraneo cerca de Barcelona, Ingenieria del Agua, 8, 15-23, (2001).

[10] Castilla R. Redondo J.M., Gamez P.J., Babiano A. Coherent Vortices and Lagrangian Dynamics in 2D Turbulence. Non-Linear Processes in Geophysics vol. 14, 139-151. (2007).

[11] Boffetta G, Celani A, Vergassola M. : Inverse energy cascade in two-dimensional turbulence: deviations from Gaussian behavior. Phys. Rev. E vol. 61:R29-32. (2000).

[12] Van Heijst G, Clercx H. Laboratory modeling of geophysical vortices. Annu. Rev. Fluid Mech. vol. 41:143-64, (2009)

[13] Chertkov M, Connaughton C, Kolokolov I, Lebedev V. Dynamics of energy condensation in two dimensional turbulence. Phys. Rev. Lett. vol. 99 : 084501 (2007)

[14] Mahjoub, O. B., Redondo, J. M., and Babiano, A.: Structure functions in complex flows, Appl. Sci. Res., vol. 59, 299-313, (1998).

[15] Redondo J.M. Vertical Microstructure and Mixing in Stratified Flows. Gavrilakis et al. (eds.), Advances in Tuerbulence VI, 605-608. Kluwer Academic Publishers (1996).

[16] Tarquis, A. M., Platonov, A., Matulka, A., Grau, J., Sekula, E., Diez, M., \& Redondo, J. M. Application of multifractal analysis to the study of SAR features and oil vol. 38:1642-1664, (2014)

[17] Redondo, J. M. and Platonov, A.: Self-similar distribution of oil spills in European coastal waters, Environ. Res. Lett., vol. 4, 14008, (2009).

[18] Redondo, J. M., Grau, J., Platonov, A., and Garzon, G.: Analisis multifractal de procesos autosimilares, Imagenes de satelite e inestabilidades baroclinas, Rev. Int. Met. Num. Calc. Dis. Ing., vol. 24, 25-48, (2008) (in Spanish).

[19] Lopez P. Tarquis A.M. Matulka A. Skadden B. Redondo J.M. Multiscaling properties on sequences of turbulent plumes images, Chaos, Solitons and Fractals, vol. 105, 128-136. (2017).

[20] Karimova, S.: Surface circulation in the Western Mediterranean shown by a synergy of satellitederived datasets, Proc. SPIE 10422, Remote Sensing of the Ocean, Sea Ice, Coastal Waters (2017).

[21] Karimova, S.: Observations of asymmetric stirring in inner and marginal seas using satellite imagery, Proc. Int. Jour. Remote Sensing, vol. 38, 6, 1642-1664 (2017). 